\title{
Applications of the $\exp (-\Phi(\xi))$-Expansion Method to Find Exact Traveling Wave Solutions of the Benney-Luke Equation in Mathematical Physics
}

\author{
S. M. Rayhanul Islam \\ Department of Mathematics, Pabna University of Science and Technology, Pabna, Bangladesh
}

Email address:

rayhanulmath@yahoo.com

To cite this article:

S. M. Rayhanul Islam. Applications of the exp(- $\Phi(\xi))$-Expansion Method to Find Exact Traveling Wave Solutions of the Benney-Luke Equation in Mathematical Physics. American Journal of Applied Mathematics. Vol. 3, No. 3, 2015, pp. 100-105. doi: $10.11648 /$ j.ajam.20150303.14

\begin{abstract}
In this article, we construct the traveling wave solutions involving parameters of nonlinear evolutions equations via the Benney-Luke equation using the $\exp (-\Phi(\xi))$-expansion method. The traveling wave solutions are expressed in terms of hyperbolic, trigonometric and rational functions. When the parameters are taken special values, the solitary waves are derived from the traveling waves. The proposed method is direct, concise elementary and effective and can be used for many other nonlinear evolutions equations.
\end{abstract}

Keywords: $\operatorname{Exp}(-\Phi(\xi))$-Expansion Method, Benney-Luke Equation, Nonlinear Evolution Equations, Traveling Wave Solution

\section{Introduction}

The nonlinear evolution equations (NLEEs) that are studied in theoretical physics, especially in the context of wave phenomena leads to various forms of wave solutions. They are solitary waves, shock wave, cnoidal waves, snoidal waves and various other types. These waves appear in various scenarios in daily real life situations. For example, solitons appear in the propagation of pulse through optical fibers while shock waves appear in the supersonic jet flow. Another example is where cnoidal waves appear in shallow water waves although an extremely rare phenomena.

In recent years, several direct methods for finding the explicit traveling wave solutions to nonlinear evolution equations (NLEEs) have been proposed such as the extended tanh-method [1], the extended tanh-function method[2], the variational iteration method[3], Exp-function method[4], the complex hyperbolic-function method[5], the extended Fexpansion method[6], the generalized Riccati equation rational expansion method[7], the Sub-ODE method[8], the $\left(G^{\prime} / G\right)$-Expansion Method[9,10,11, 12, 13,14], the ansatz method[15], Outline of the Tanh-Coth Method [16], Cauchy Problem [17,18,19] and so on.

The aim of this paper was to apply the $\exp (-\Phi(\xi))$ expansion method [20,21] to construct the new exact traveling wave solutions for nonlinear evolution equations in mathematical physics via the Benney-Luke equation.The organization of the paper is as follows: In section 2, a description of the main steps of the $\exp (-\Phi(\xi))$-expansion method for finding the traveling wave solutions of nonlinear equations are given. In section 3, we apply this method to the nonlinear Benney-Luke equation; in section 4 we discuss the results and discussion of the traveling wave solutions. Finally concluding remarks are presented in section 5.

\section{Description of the $\exp (-\Phi(\xi))-$ Expansion Method}

In this section, we will describe the algorithm of the exp($\Phi(\xi))$-expansion method for finding traveling wave solutions of nonlinear evolution equations. Suppose that a non linear equation in two independent variables $x$ and $t$ is given by,

$$
P\left(u, u_{t}, u_{x}, u_{t t}, u_{x x}, u_{x t}, \ldots \ldots \ldots . .\right)=0,
$$$$
x \in R, t>0(2.1)
$$

where $u(\xi)=u(x, t)$ is an unknown function, $\mathrm{P}$ is a polynomial of $\mathrm{u}(\mathrm{x}, \mathrm{t})$ and its partial derivatives in which the highest order derivatives and non linear terms are involved. 
Step 1.Combining the independent variables $\mathrm{x}$ and $\mathrm{t}$ into one variables $\xi=x \pm V t$, we suppose that

$$
u(x, t)=u(\xi) \quad \xi=x \pm V t
$$

where $\omega \in R-\{0\}$ is the velocity of relative wave mode.

The traveling wave transformation equation (2.2) permits us to reduce equation (2.1) to the following ordinary differential equation (ODE)

$$
F\left(u, u^{\prime}, u^{\prime \prime}, \ldots \ldots \ldots\right)=0
$$

Where $F$ is a polynomial in $u(\xi)$ and its derivatives, whereas $u^{\prime}(\xi)=\frac{d u}{d \xi}, u^{\prime \prime}(\xi)=\frac{d^{2} u}{d \xi^{2}}$, and so on.

Step 2. Suppose the traveling wave solution of Eq. (2.3) can be expressed as follows:

$$
u(\xi)=\sum_{i=0}^{N} A_{i}(\exp (-\Phi(\xi)))^{i}
$$

Where $A_{i}(0 \leq i \leq N)$ are constants to be determined, such that $A_{N} \neq 0$, and $\Phi=\Phi(\xi)$ satisfies the following ODE,

$$
\Phi^{\prime}(\xi)=\exp (-\Phi(\xi))+\mu \exp (\Phi(\xi))+\lambda
$$

Eq. (2.5) gives the following solutions:

Family 1: When $\lambda^{2}-4 \mu>0, \mu \neq 0$,

$$
\begin{gathered}
\Phi(\xi)=\ln \left(\frac{-\sqrt{\left(\lambda^{2}-4 \mu\right)} \tanh \left(\frac{\sqrt{\left(\lambda^{2}-4 \mu\right)}}{2}(\xi+k)\right)-\lambda}{2 \mu}\right) \\
\Phi(\xi)=\ln \left(\frac{-\sqrt{\left(\lambda^{2}-4 \mu\right)} \operatorname{coth}\left(\frac{\sqrt{\left(\lambda^{2}-4 \mu\right)}}{2}(\xi+k)\right)-\lambda}{2 \mu}\right)
\end{gathered}
$$

Family 2: When $\lambda^{2}-4 \mu<0, \mu \neq 0$,

$$
\begin{gathered}
\Phi(\xi)=\ln \left(\frac{\sqrt{\left(4 \mu-\lambda^{2}\right)} \tan \left(\frac{\sqrt{\left(4 \mu-\lambda^{2}\right)}}{2}(\xi+k)\right)-\lambda}{2 \mu}\right) \\
\Phi(\xi)=\ln \left(\frac{\sqrt{\left(4 \mu-\lambda^{2}\right)} \cot \left(\frac{\sqrt{\left(4 \mu-\lambda^{2}\right)}}{2}(\xi+k)\right)-\lambda}{2 \mu}\right)
\end{gathered}
$$

Family 3 : When $\lambda^{2}-4 \mu>0, \mu=0, \lambda \neq 0$,

$$
\Phi(\xi)=-\ln \left(\frac{\lambda}{\exp (\lambda(\xi+k))-1}\right)
$$

Family 4: When $\lambda^{2}-4 \mu=0, \mu \neq 0, \lambda \neq 0$,

$$
\Phi(\xi)=\ln \left(-\frac{2(\lambda(\xi+k)+2)}{\left.\lambda^{2}(\xi+k)\right)}\right)
$$

Family 5: When $\lambda^{2}-4 \mu=0, \mu=0, \lambda=0$,

$$
\Phi(\xi)=\ln (\xi+k)
$$

where $\mathrm{k}$ is an arbitrary constant and $A_{N}, \cdots \cdots, V, \lambda, \mu$ are constants to be determined later, $A_{N} \neq 0$, the positive integer $N$ can be determined by considering the homogeneous balance between the highest order derivatives and the nonlinear terms appearing in Eq. (2.3).

Step 3. We substitute Eq. (2.4) into (2.3) and then we account the function $\exp (-\Phi(\xi))$. As a result of this substitution, we get a polynomial of $\exp (-\Phi(\xi))$. We equate all the coefficients of same power of $\exp (-\Phi(\xi))$ are equal to zero. This procedure yields a system of algebraic equations whichever can be solved to find $A_{1}, A_{2}, \cdots \cdots, V, \lambda, \mu$. Substituting the values of $A_{1}, A_{2}, \cdots \cdots, V, \lambda, \mu$ into Eq. (2.4) along with general solutions of Eq. (2.5) completes the determination of the solution of Eq. (2.1).

\section{Application}

In this section, we will make use of the $\exp (-\Phi(\xi))$ expansion method to find the exact solitary wave solutions to the Benney-Luke equation. Let us consider the BenneyLuke equation in the form

$$
u_{t t}-u_{x x}+\alpha u_{x x x x}-\beta u_{x x t t}+u_{t} u_{x x}+2 u_{x} u_{x t}=0
$$

Where $\alpha$ and $\beta$ are positive number such that $\alpha-\beta=\sigma-1 / 3$ is a Sobolev type equation and studied for a very long time. The dimensionless parameter $\sigma$ is named the Bond number, which captures the effects of surface tension and gravity force and is a formally valid approximation for describing two-way water wave propagation in presence of surface tension [22].

Using the traveling wave variable $\xi=x-V t$, Eq. (3.1) converts into the following $\operatorname{ODE}$ for $u(x, t)=u(\xi)$,

$$
\left(V^{2}-1\right) u^{\prime \prime}+\left(\alpha-\beta V^{2}\right) u^{(4)}-3 V u^{\prime} u^{\prime \prime}=0
$$

Eq. (3.2) is integrable, therefore integrating with respect to $\xi$ once and choosing the integration constant to zero, we obtain

$$
\left(V^{2}-1\right) u^{\prime}+\left(\alpha-\beta V^{2}\right) u^{3}-\frac{3}{2} V u^{\prime 2}=0
$$


Where, primes denote the differentiation with regard to $\xi$. By balancing the highest order term $u^{\prime 2}$ and nonlinear term of the highest order $u^{3}$, we obtain $N=1$. Therefore, the $\exp (-\Phi(\xi))$-expansion method admits the solution of the Eq.(3.3) in the form

$$
u(\xi)=A_{0}+A_{1}(\exp (-\varphi(\xi))), \quad A_{1} \neq 0
$$

By substituting the Eq.(2.5) and (3.4) into the Eq.(3.3) and equating the coefficient of $(\exp (-\Phi(\xi)))^{i}$, are equal to zero, yielding a set of algebraic equations as follows:

$$
\begin{gathered}
-A_{1} V^{2} \mu-\alpha A_{1} \mu \lambda^{2}+2 \beta V^{2} A_{1} \mu^{2}-\frac{3}{2} V A_{1}^{2} \mu^{2}+\beta V^{2} A_{1} \mu \lambda^{2}+A_{1} \mu-2 \alpha A_{1} \mu^{2}=0 \\
-8 \alpha A_{1} \mu \lambda+A_{1} \lambda-\alpha A_{1} \lambda^{3}+\beta V^{2} A_{1} \lambda^{3}-3 V A_{1}^{2} \mu \lambda+8 \beta V^{2} A_{1} \mu \lambda-A_{1} V^{2} \lambda=0 \\
-8 \alpha A_{1} \mu-A_{1} V^{2}+7 \beta V^{2} A_{1} \lambda^{2}-7 \alpha A_{1} \lambda^{2}-3 V A_{1}^{2} \mu+A_{1}-\frac{3}{2} V A_{1}^{2} \lambda^{2}+8 \beta V^{2} A_{1} \mu=0 \\
-3 V A_{1}^{2} \lambda+12 \beta V^{2} A_{1} \lambda-12 \alpha A_{1} \lambda=0 \\
-6 \alpha A_{1}-\frac{3}{2} V A_{1}^{2}+6 \beta V^{2} A_{1}=0 \quad(3.8) \quad \xi=x \pm \frac{\sqrt{\left(1+4 \beta \mu-\beta \lambda^{2}\right)(1+4}}{1+4 \beta \mu-\beta \lambda^{2}} \\
Q=\frac{8(\alpha-\beta) \mu}{\sqrt{(1+4 \beta \mu}}
\end{gathered}
$$$$
-8 \alpha A_{1} \mu \lambda+A_{1} \lambda-\alpha A_{1} \lambda^{3}+\beta V^{2} A_{1} \lambda^{3}-3 V A_{1}^{2} \mu \lambda+8 \beta V^{2} A_{1} \mu \lambda-A_{1} V^{2} \lambda=0
$$

Solving the algebraic Eq. (3.5) to (3.9), we obtain a set of solution as follows:

$$
\begin{aligned}
& V= \pm \frac{\sqrt{\left(1+4 \beta \mu-\beta \lambda^{2}\right)\left(1+4 \alpha \mu-\alpha \lambda^{2}\right)}}{1+4 \beta \mu-\beta \lambda^{2}}, \quad A_{0}=A_{0}, \\
& A_{1}= \pm \frac{4(\alpha-\beta)}{\sqrt{\left(1+4 \beta \mu-\beta \lambda^{2}\right)\left(1+4 \alpha \mu-\alpha \lambda^{2}\right)}}
\end{aligned}
$$

Where $\lambda$ and $\mu$ are arbitrary constants.

By substituting Eq.(3.10)into Eq.(3.4), we have

$u(\xi)=A_{0} \pm \frac{4(\alpha-\beta)}{\sqrt{\left(1+4 \beta \mu-\beta \lambda^{2}\right)\left(1+4 \alpha \mu-\alpha \lambda^{2}\right)}}(\exp (-\varphi(\xi)))$

Where

$$
\xi=x \pm \frac{\sqrt{\left(1+4 \beta \mu-\beta \lambda^{2}\right)\left(1+4 \alpha \mu-\alpha \lambda^{2}\right)}}{1+4 \beta \mu-\beta \lambda^{2}} t
$$

Now substituting Eq. (2.6) to Eq. (2.12) into Eq. (3.11) respectively, the traveling wave solutions of the Eq.(3.1) are obtained as follows:

Family 1: When $\lambda^{2}-4 \mu>0, \mu \neq 0$,

$$
\begin{aligned}
& u_{1,2}(x, t)=A_{0} \pm \frac{Q}{\left(-\sqrt{\lambda^{2}-4 \mu} \tanh \left(\frac{\sqrt{\lambda^{2}-4 \mu}}{2}(\xi+k)\right)-\lambda\right)}, \\
& u_{3,4}(x, t)=A_{0} \pm \frac{Q}{\left(-\sqrt{\lambda^{2}-4 \mu} \operatorname{coth}\left(\frac{\sqrt{\lambda^{2}-4 \mu}}{2}(\xi+k)\right)-\lambda\right)},
\end{aligned}
$$

$$
\begin{aligned}
& \xi=x \pm \frac{\sqrt{\left(1+4 \beta \mu-\beta \lambda^{2}\right)\left(1+4 \alpha \mu-\alpha \lambda^{2}\right)}}{1+4 \beta \mu-\beta \lambda^{2}} t, \\
& Q=\frac{8(\alpha-\beta) \mu}{\sqrt{\left(1+4 \beta \mu-\beta \lambda^{2}\right)\left(1+4 \alpha \mu-\alpha \lambda^{2}\right)}}
\end{aligned}
$$

Family 2: When $\lambda^{2}-4 \mu<0, \mu \neq 0$,

$$
\begin{aligned}
& u_{5,6}(x, t)=A_{0} \pm \frac{Q}{\left(\sqrt{4 \mu-\lambda^{2}} \tan \left(\frac{\sqrt{4 \mu-\lambda^{2}}}{2}(\xi+k)\right)-\lambda\right)} \\
& u_{7,8}(x, t)=A_{0} \pm \frac{Q}{\left(\sqrt{4 \mu-\lambda^{2}} \cot \left(\frac{\sqrt{4 \mu-\lambda^{2}}}{2}(\xi+k)\right)-\lambda\right)}
\end{aligned}
$$

Where

$$
\begin{aligned}
& \xi=x \pm \frac{\sqrt{\left(1+4 \beta \mu-\beta \lambda^{2}\right)\left(1+4 \alpha \mu-\alpha \lambda^{2}\right)}}{1+4 \beta \mu-\beta \lambda^{2}} t \\
& Q=\frac{8(\alpha-\beta) \mu}{\sqrt{\left(1+4 \beta \mu-\beta \lambda^{2}\right)\left(1+4 \alpha \mu-\alpha \lambda^{2}\right)}}
\end{aligned}
$$

Family 3 : When $\lambda^{2}-4 \mu>0, \mu=0, \lambda \neq 0$,

$$
u_{9,10}(x, t)=A_{0} \pm Q(\exp (\lambda(\xi+k))-1),
$$

Where

$$
\begin{aligned}
& \xi=x \pm \frac{\sqrt{\left(1-\beta \lambda^{2}\right)\left(1-\alpha \lambda^{2}\right)}}{1-\beta \lambda^{2}} t \\
& Q=\frac{4(\alpha-\beta) \lambda}{\sqrt{\left(1-\beta \lambda^{2}\right)\left(1-\alpha \lambda^{2}\right)}}
\end{aligned}
$$

Family 4: When $\lambda^{2}-4 \mu=0, \mu \neq 0, \lambda \neq 0$, 


$$
u_{11,12}(x, t)=A_{0} \pm \frac{2(\alpha-\beta) \lambda^{2}(\xi+k)}{\lambda(\xi+k)+2}
$$

Where $\xi=x \pm t$

Family 5: When $\lambda^{2}-4 \mu=0, \mu=0, \lambda=0$,

$$
u_{13,14}(x, t)=A_{0} \pm \frac{4(\alpha-\beta)}{\xi+k}
$$

Where $\xi=x \pm t$

\section{Results and Discussion}

In this section, we describe the physical explanation and graphical representation of the solutions of the Benney-Luke equation.

\subsection{Explanation}

The solution $u(x, t)$ to the solitary wave Eq.(3.1) play an important role for describing different types of wave propagation of pulses through optical fibers while shock waves appear in the supersonic jet flow. The Eq.(3.1) is given not only more new multiple explicit solutions but also many types of exact traveling wave solutions. The exact traveling wave solutions are obtained from the explicit solutions by choosing the particular value of the physical parameters. So we can appropriate values of the parameters to obtained exact solutions. There are various types of traveling wave solutions that are particular interest in solitary wave theory. In this research work, some important traveling wave solutions are described and presented graphically.

The solution $u_{1}(x, t)$ is presented the kink type solitonsolution. Kink solitons are rise from one asymptotic state at $\xi \rightarrow-\infty$ to another asymptotic state at $\xi \rightarrow+\infty$. These solitons are referred to as topological solitons. The Fig. 1 has been shown the shape of the solution $u_{1}(x, t)$ for $\alpha=6, \beta=3, \lambda=8, \mu=1.5, A_{0}=1, k=0.5$ within the interval $-3 \leq x, t \leq 3$. The solution $u_{5}(x, t)$ is presented the periodic soliton solution for the various values of the physical parameters. The Fig. 2 has been shown the shape of the solution $u_{5}(x, t)$ for $\alpha=7, \beta=1, \lambda=-1, \mu=1, A_{0}=0 k=2$ within the interval $-10 \leq x, t \leq 10$. For the values of $\alpha=5, \beta=2, \lambda=1, \mu=0, A_{0}=3, k=0$ within the interval $-10 \leq x, t \leq 10$, the solution $u_{9}(x, t)$ is presented the soliton profile which is shows in Fig. 3. The solution $u_{11}(x, t)$ is also presented the singular kink type soliton solution which is shows in Fig. 4 for $\alpha=8, \beta=3, \lambda=1, k=0.8, A_{0}=0$ within the interval $-10 \leq x, t \leq 10$. Finally, Fig. 5 shows an exact singular kink type solitary wave profile corresponding to $u_{13}(x, t)$ with fixed parameters $\alpha=7, \beta=3, k=2, A_{0}=2$ within the interval $-10 \leq x, t \leq 10$.

\subsection{Graphical Representation}

In this sub section, the graphical representations of the solutions are given below in the figures (Fig. 1-5) with the aid of mathematical software Maple 13.

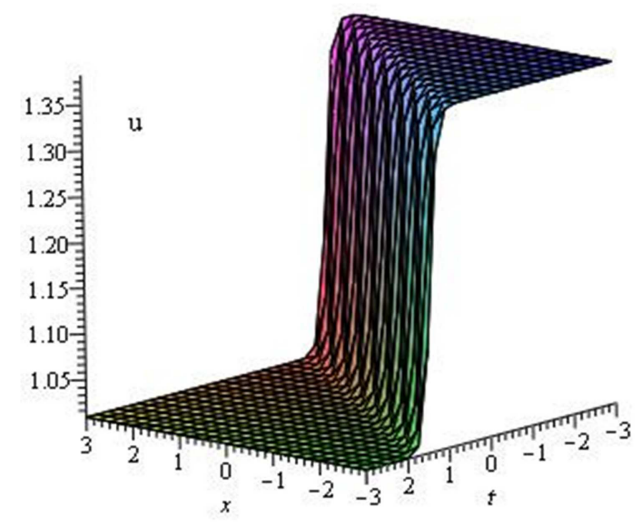

Fig. 1. Kink type soliton profile, Shape of $u_{1}(x, t)$ when $\alpha=6, \beta=3, \lambda=8, \mu=1.5 \quad A_{0}=1, k=0.5$ with $-3 \leq x, t \leq 3$

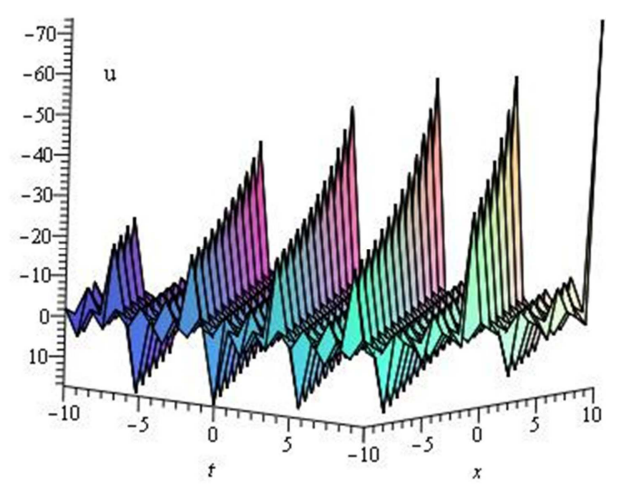

Fig. 2. Periodic type soliton profile, Shape of $u_{5}(x, t)$ when $\alpha=7, \beta=1, \lambda=-1, \mu=1 \quad A_{0}=0, k=2$ with $-10 \leq x, t \leq 10$.

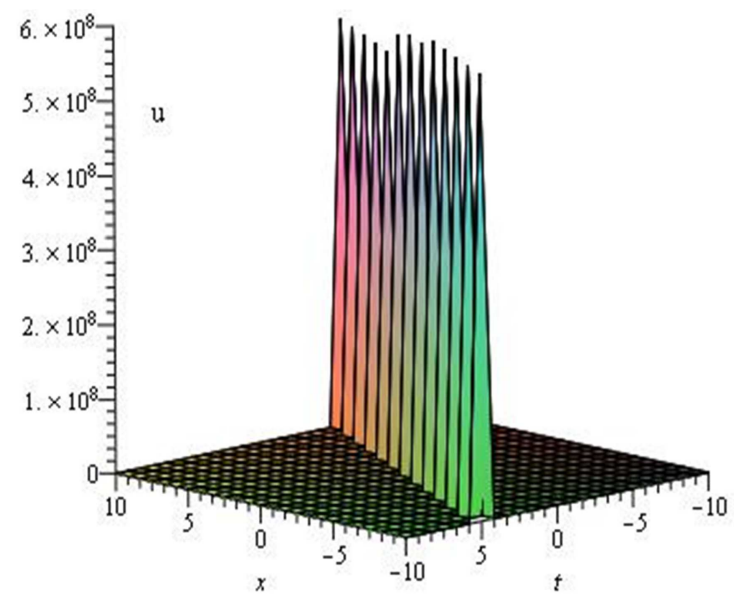

Fig. 3. soliton profile, Shape of $u_{9}(x, t)$ when $\alpha=5, \beta=2, \lambda=1, \mu=0 \quad A_{0}=3, k=0$ with $-10 \leq x, t \leq 10$. 


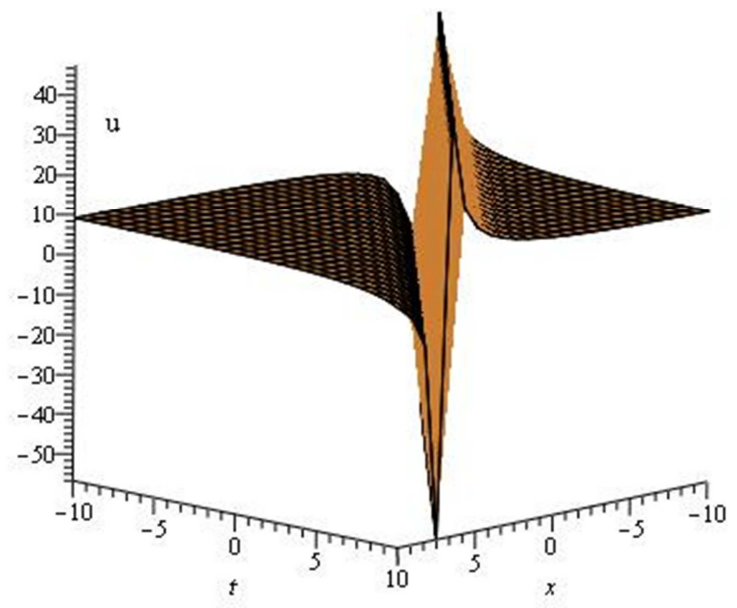

Fig. 4. Singular kink typesoliton profile, Shape of $u_{11}(x, t)$ when $\alpha=8, \beta=3, \lambda=1 \quad A_{0}=0, k=0.8$ with $-10 \leq x, t \leq 10$.

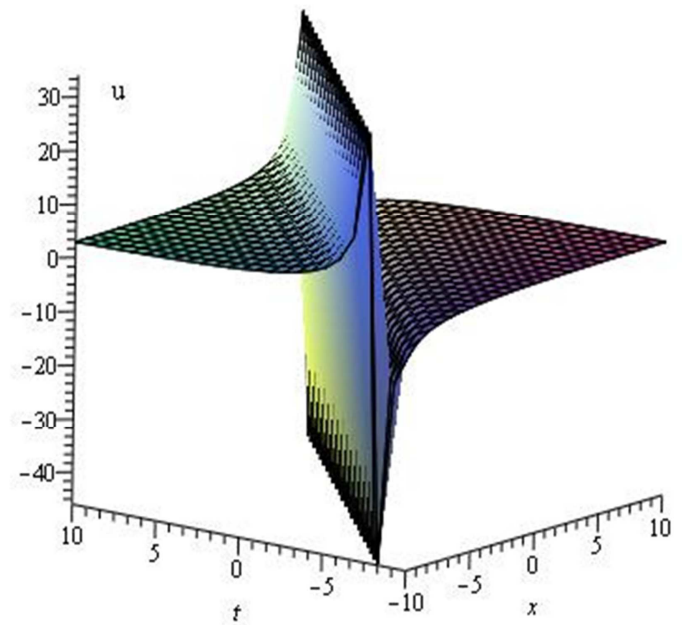

Fig. 5. Singular kink typesoliton profile, Shape of $u_{13}(x, t)$ when $\alpha=7, \beta=3, A_{0}=2, k=2$ with $-10 \leq x, t \leq 10$.

\section{Conclusion}

In this paper, the $\exp (-\Phi(\xi))$-expansion method has been successfully applied to construct new traveling wave solutions for the Benney-Luke equation. This study shows that the $\exp (-\Phi(\xi))$-expansion method is quite efficient and practically well suited for use in finding exact solutions for the problems considered here. Being concise and effective, the $\exp (-\Phi(\xi))$-expansion method can also be used to many other nonlinear equations.

\section{References}

[1] M. A. Abdou, "The extended tanh- method and its applications for solving nonlinear physical models," Appl. Math. Comput., 190, 988-996, 2007.

[2] E.G. Fan, "Extended tanh-function method and its applications to nonlinear equations," Physics Letter A, 277, 212-218, 2000.
[3] J.H. He, "Variational iteration method for delay differential equations,"Commun. Nonlinear Sci. Numer. Simul., 2, 235-6, 1997.

[4] J. H. He, X. H. Wu, "Exp-function method for nonlinear wave equations," Chaos Solitons Fractals, 30, 700-708, 2006.

[5] C. Bai, H. Zhao, "Complex hyperbolic-function method and its applications to nonlinear equations," Physics Letter A, 355 22-30, 2006.

[6] M. L. Wang, X. Li, "Extended F- expansion and periodic wave solutions for the generalized Zakharov equations," Physics Letter A, 343, 48-54, 2005.

[7] Z. Wang, H. Q. Zhang, "A new generalized Riccati equation rational expansion method to a class of nonlinear evolution equation with nonlinear terms of any order," Appl. Math. Comput., 186, 693-704, 2007.

[8] M. L. Wang, X. Z. Li, J. L. Zhang, "Sub-ODE method and solitary wave solutions for higher ordernonlinear Schrodinger equation," Phys. Lett. A, 363, 96-101, 2007.

[9] N.Taghizade, A. Neirameh, "The solution of TRLW and Gardner Equations by the $\left(\mathrm{G}^{\prime} / \mathrm{G}\right)$-Expansion Method. International Journal of Nonlinear Science, 9, 305-310, 2010.

[10] A. Bekir, "Application of the $\left(\mathrm{G}^{\prime} / \mathrm{G}\right)$ - expansion method for nonlinear evolution equations," 372, 3400-3406, 2008.

[11] E. M. E. Zayed, "Traveling wave solutions for higher dimensional nonlinear evolution equations using the $\left(\mathrm{G}^{\prime} / \mathrm{G}\right)$ expansion method,” J. Appl. Math. Inform., 28, 383-395, 2010.

[12] E. M. E. Zayed, K. A. Gepreel, "The $\left(\mathrm{G}^{\prime} / \mathrm{G}\right)$ - expansion method for finding the traveling wave solutions of nonlinear partial differential equations in mathematical physics," J. Math. Phys., 50, 013502-14, 2009.

[13] M. Mirzazadeh, M. Eslami, A. Biswas, "Soliton solutions of the generalized Klein-Gordon equation by the $\left(\mathrm{G}^{\prime} / \mathrm{G}\right)$ expansion method," Comput. Appl. Math., 33, 831-839, 2014.

[14] Z. Yan, H. Zhang, "New explicit solitary wave solutions and periodic wave solutions for WhithamBroer-Kaup equation in shallow water," Phys. Lett. A, 285, 355-362, 2001.

[15] H. Triki, A. Yildirim, T. Hayat, O. M. Aldossary, A. Biswas, "Shock wave solution of the Benney-Luke equation," Rom. J. Phys., 57, 1029-34, 2012.

[16] O. Gozukizil, S. Akcagil, "Traveling wave solutions to the Benney-Luke and the higher order improve Boussinesq equations of Soobelev type," Abstract and applied analysis, volume 2012, Article ID 890574, 10 pages. DOI: $10.1155 / 2012 / 890574$.

[17] A. Gonz'alez N., "The Cauchy problem for Benney-Luke and generalized Benney-Luke equations," Differential and Integral Equations, 20, 1341-1362, 2007.

[18] J. R. Quintero, "A remark on the Cauchy problem for the generalized Benney-Luke equation,'Differential and Integral Equations, 21, 859-890, 2008.

[19] S. Wang, G. Xu, and G. Chen, "Cauchy problem for the generalized Benney-Luke equation," Journal of Mathematical Physics, vol. 48, no. 7, Article ID 073521, 2007. 
[20] S. M. R. Islam,K. Khan, M. A. Akbar, "Exact solutions of unsteady Korteweg-de vries and time regularized long wave equations," Springer plus, 4, 124, 2015. DOI 10.1186/s40064-015-0893-y.

[21] S. M. R. Islam, K. Khan, M. A. Akbar, "Study of exp(- $\Phi(\xi))-$ expansion method for solving nonlinear partial differential equations," British Journal of Mathematics \& Computer Science, 5, 397-407, 2015.
[22] J. R. Quintero, J. C. Munoz Grajales, "Instability of solitary waves for a generalized Benney-Luke equation," Nonlinear Analysis, Theory, Methods and Applications, 68,3009-3033, 2008. 